\title{
A rare case of Mitochondrial Fatty Acid Oxidation Defect- Systemic Primary Carnitine Deficiency
}

\author{
Garg $M^{1}$, Dhruw $S^{2}$, Mangal D. $K^{3}$, Singhal $C^{4}$, Khan $K^{5}$ \\ ${ }^{1}$ Dr Manisha Garg, Senior Resident, Department of Paediatrics, SMS Medical College, Jaipur, India, ${ }^{2}$ Dr Sneha Dhruw, \\ Senior Resident, Department of Paediatrics, SMS Medical College, Jaipur, India, ${ }^{3}$ Dr Dhananjay Kumar Mangal, Senior \\ Consultant \& Director of Babylon Hospital, Jaipur, India, ${ }^{4}$ Dr Chanchal Singhal, Consultant of Babylon Hospital, Jaipur, \\ India, ${ }^{5}$ Dr Khurshida Khan, Senior Resident, Department of Paediatrics, SMS Medical College, Jaipur, India.
}

Address for Correspondence: Dr. Manisha Garg, D/o Dr. S. N. Garg, House No. 28, M.P. Colony, Mantown, Sawai Madhopur, Rajasthan, India. Email: drgargmanisha@gmail.com

\begin{abstract}
Systemic primary carnitine deficiency (SPCD) also known as, carnitine transporter deficiency (CTD) is an inborn error of fatty acid transport caused by a defect in the transporter responsible for moving carnitine across the plasma membrane, leading to a variety of symptoms such as chronic muscle weakness, cardiomyopathy and hypoglycaemia and liver dysfunction. The first suspicion of SPCD in a patient with a non-specific presentation is an extremely low plasma carnitine level, confirmed by demonstrating reduced carnitine transport in skin fibroblasts from the patient. Treatment for SPCD involves high dose carnitine supplementation, which must be continued for life. We report a case of Systemic primary carnitine deficiency with classical clinical \& laboratory characteristics.
\end{abstract}

Key words: Primary carnitine deficiency, Carnitine, Fatty acid oxidation.

\section{Introduction}

Carnitine is a naturally occurring hydrophilic amino acid derivative, plays an essential role in the transfer of long-chain fatty acids into the mitochondria for betaoxidation $[1,2]$. When carnitine cannot be transported into tissues, fatty acid oxidation is impaired, leading to a variety of symptoms such as chronic muscle weakness, cardiomyopathy, hypoglycemia and liver dysfunction. Acute episode preceded by metabolic stress such as extended fasting, infections or vomiting.

Carnitine deficiency may be primary or secondary. Systemic primary carnitine deficiency, (SPCD) also known as carnitine uptake defect, carnitine transporter deficiency (CTD) or systemic carnitine deficiency is an inborn error of fatty acid transport caused by a defect in the transporter responsible for moving carnitine across the plasma membrane. The specific transporter involved with SPCD is OCTN2, coded for by the SLC22A5 gene located on chromosome 5[3].

SPCD is inherited in an autosomal recessive manner, with mutated alleles coming from both parents $[4,5]$. Primary carnitine deficiency has a frequency of about 1:40,000 newborns in Japan [6] and 1:37,000-1:100,000 newborns in Australia [7]. In the USA and Europe, the frequency of primary carnitine deficiency has not been defined, but from the reported cases, it seems similar to that in Japan.

\section{Case Report}

A 9 month old female child first born of a 3 degree consanguineous marriage was brought to us with complains of fever and vomiting for 10 days, yellow discolouration of eyes, unable to sit and hold neck, abnormal movements and altered level of sensorium for 8 days and swelling over both lower limbs for 3 days. There was no history of any drug ingestion.

Manuscript received: $5^{\text {th }}$ October 2016

Reviewed: $16^{\text {th }}$ October 2016

Author Corrected; $28^{\text {th }}$ October 2016

Accepted for Publication: $15^{\text {th }}$ November 2016 
Development was appropriate for age before illness. Antenatal period was normal with birth weight of $3.5 \mathrm{~kg}$. There was no similar history in family. Growth of child was normal as anthropometric measurements were within normal range. Examination showed pallor, icterus, pitting oedema over both lower limbs with no dysmorphic features. Systemic examination showed firm liver with sharp margined, liver span was $8 \mathrm{~cm}$, abnormal movements involving both upper limbs and neck and generalised hypotonia, with absent deep tendon reflexes, rest of the examination was normal.

Blood investigations revealed anemia (haemoglobin-7.8 mg/dl ), raised alanine amino-transferase and aspartate aminotransferase levels were $180 \mathrm{U} / \mathrm{L}$ and $210 \mathrm{U} / \mathrm{L}$ respectively, elevated serum billirubin (total-7.3 mg/dl ,direct $-4.1 \mathrm{mg} / \mathrm{dl}$ ), elevated gama glutamil transpeptidase $-379 \mathrm{Iu} / \mathrm{L}$, elevated C-reactive protein-40 Mg/L and low blood sugar-46 mg/dl. Renal function tests were within normal range. Work up for, Hepatitis viral marker, Malaria antigen and Leptospira were negative. Direct Coombs test was negative. Urine examination showed absence of ketone bodies. Serum Creatine kinase activity was within normal range. Ultrasonography of abdomen showed bright enlarged liver with minimal ascites. Magnetic resonance imaging (MRI) and Magnetic resonance spectroscopy (MRS) findings showed symmetrical hyperintensities seen on $\mathrm{T} 2 \mathrm{~W}$ scans involving periventricular, deep subcortical white matter of bilateral cerebral hemisphere, bilateral thalami and white matter of bilateral cerebral hemispheres. Affected area shows restriction on diffusion weighted scans- possibly due to mitochondrial disorder and MRS showed reduced NAA with elevated Choline; mildly elevated lactate peak seen at $1.3 \mathrm{ppm}$. N-acetylaspartate to creatine ratio (NAA/Cr) are reduced. Gas chromatography-mass spectrometry (GC-MS) of urine showed elevated lactic acid, pyruvic acid and presence of fumaric acid.

Then we planned carnitine /acyl carnitine profile in blood and plasma amino acids. Tendom mass spectroscopy of blood showed very low free $(2.35 \mathrm{umol} / \mathrm{L})$ and low total carnitine $(8.37 \mathrm{umol} / \mathrm{L})$ suggestive of severe carnitine deficiency with normal levels of amino acids. A diagnosis of Systemic primary carnitine deficiency was made. Child was commenced on treatment with IV 10\% dextrose and Oral L-carnitine therapy was started via nasogastric route. As the patient did not survive so we could not do mutational analysis.

\section{Discussion}

Carnitine ( $\beta$-hydroxy- $\gamma$-trimethylammonium butyrate) is a hydrophilic molecule that plays an essential role in the transfer of long-chain fatty acids inside mitochondria for $\beta$ oxidation $[1,2]$. Carnitine binds acyl residues and helps in their elimination, decreasing the number of acyl residues conjugated with coenzyme A $(\mathrm{CoA})$ and increasing the ratio between free and acylated CoA [8]. During periods of fasting, fatty acids turn into the predominant substrate for energy production via oxidation in the liver, cardiac muscle, and skeletal muscle. If fatty acid oxidation is defective, fat cannot be utilized, glucose is consumed without regeneration via gluconeogenesis and there is a drop in glucose levels (hypoglycemia), and the production of ketone bodies (which are used by the brain) is also impaired.

Carnitine deficiency may be primary or secondary. Primary carnitine deficiency is an autosomal recessive disorder of fatty acid oxidation due to the lack of functional OCTN2 carnitine transporters [3]. The lack of the plasma membrane carnitine transporter results in urinary carnitine wasting, low serum carnitine levels, and decreased intracellular carnitine accumulation. Patients with primary carnitine deficiency lose most
(90-95\%) of the filtered carnitine in urine and their heterozygous parents lose 2 to 3 times the normal amount, explaining their mildly reduced plasma carnitine levels [9]. Secondary carnitine deficiency, which manifests with a decrease of carnitine levels in plasma or tissues, may be associated with genetically determined metabolic conditions, acquired medical conditions, or iatrogenic states.

One classic initial presentation of primary carnitine deficiency is hypoketotic hypoglycemic encephalopathy, accompanied by hepatomegaly, elevated liver transaminases, and hyperammonemia. Cardiomyopathy is the other classic presentation (affecting older children), Muscle weakness, the third manifestation of the disease. Key to the diagnosis is the measurement of plasma carnitine levels. Free and acylated carnitine are extremely reduced (free carnitine $<5 \mu \mathrm{M}$ ) and urine organic acids do not show any consistent anomaly, although a non-specific dicarboxylic aciduria has been reported. Diagnosis is confirmed by demonstrating reduced carnitine transport in skin fibroblasts from the patient. This is usually reduced below $10 \%$ of the value of matched controls [9]. 
Primary carnitine deficiency can be identified in infants by expanded newborn screening using tandem mass spectrometry by detection of low levels of free carnitine (C0). The only anomaly on the acylcarnitine profile is a low level of free carnitine and all acylcarnitine species. Carnitine is transferred by the placenta to the growing fetus and plasma levels decrease rapidly after birth [7].

Primary carnitine deficiency should be differentiated from other causes of carnitine deficiency. These include a number of organic acidemias, defects of fatty acid oxidation and of the carnitine cycle [9]. In all these disorders, analysis of urine organic acids, plasma amino acids and acylcarnitine profile, in conjunction with the clinical presentation, allows a definitive diagnosis.

Medical therapy with oral carnitine in primary carnitine deficiency improves fasting ketogenesis, cardiac function, growth, and cognitive performance. Actively avoid periods of fasting in these patients.

\section{Conclusion}

Our case provides a very good insight into the approach to the diagnosis of Systemic primary carnitine deficiency. In most locations with expanded newborn screening, SPCD can be identified and treated shortly after birth. Treatment with high doses of carnitine supplementation is effective, but needs to be rigorously maintained for life.

Funding: No funding sources

Conflict of Interest: None declared

Ethical approval: Not required

\section{References}

1. Longo N, Amat di San Filippo C, Pasquali M. Disorders of carnitine transport and the carnitine cycle. Am J Med Genet C Semin Med Genet. 2006; 142 $\mathrm{C}(2): 77-85$.
2. Roe C, Ding J. Mitochondrial fatty acid oxidation disorders. In: Scriver C, Beaudet A, Sly W, Valle D, editors. The Metabolic and Molecular Bases of Inherited Disease. 8 McGraw-Hill; New York: 2001. pp. 2297-2326.

3. Shoji Y, Koizumi A, Kayo T, Ohata T, Takahashi T, Harada K, Takada G. Evidence for linkage of human primary systemic carnitine deficiency with D5S436: a novel gene locus on chromosome 5q. Am J Hum Genet. 1998 Jul;63(1):101-8.

4. Waber, L. J., Valle, D., Neill, C., DiMauro, S., Shug, A. Carnitine deficiency presenting as familial cardiomyopathy: a treatable defect in carnitine transport. J. Pediat. 101: 700-705, 1982.

5. Treem WR, Stanley CA, Finegold DN, Hale DE, Coates PM. Primary carnitine deficiency due to a failure of carnitine transport in kidney, muscle, and fibroblasts. N Engl J Med.1988 Nov17;319(20):1331-6.

6. Koizumi A et al., Genetic epidemiology of the carnitine transporter OCTN2 gene in a Japanese population and phenotypic characterization in Japanese pedigrees with primary systemic carnitine deficiency. Hum Mol Genet. 1999;8:2247-2254.

7. Wilcken B, Wiley V, Sim KG, Carpenter K. Carnitine transporter defect diagnosed by newborn screening with electrospray tandem mass spectrometry. J Pediatr. 2001;138:581-584.

8. Bieber LL. Carnitine. Annu Rev Biochem. 1988; 57:261-83.

9. Scaglia F, Wang Y, Singh RH, Dembure PP, Pasquali M, Fernhoff PM, Longo N. Defective urinary carnitine transport in heterozygotes for primary carnitine deficiency. Genet Med. 1998;1:34-9.

\section{How to cite this article?}

Garg M, Dhruw S, Mangal D. K, Singhal C, Khan K. A rare case of Mitochondrial Fatty Acid Oxidation DefectSystemic Primary Carnitine Deficiency. Int. J Pediatr Res. 2016;3(11):802-804.doi:10.17511/ijpr.2016.i11.05. 